\author{
Zong-Gang MA, PhD \\ School of Finance \\ Guangdong University of Finance and Economics \\ Guangzhou, PR China \\ E-mail: mazonggang@gdufe.edu.cn \\ Shi-Song XIAO, PhD \\ School of Business Administration \\ Hunan University, Changsha, PR China \\ E-mail:shsiao@yeah.net
}

\title{
CLOSED FORM VALUATION OF VULNERABLE EUROPEAN OPTIONS WITH STOCHASTIC CREDIT SPREADS
}

\begin{abstract}
This paper develops a two-factor valuation model of vulnerable European options incorporating a stochastic credit spread, which is formulated as a mean-reverting Ornstein-Uhlenbeck stochastic process. Furthermore, we exploit Mellin transform techniques to derive a closed-form solution for vulnerable European options in Black-Scholes model. The formula is simply provided with standard normal cumulative distribution function so that the pricing and hedging of the options can be computed very accurately and rapidly. Numerical experiments demonstrate that how credit risks depends only on the prices of bonds that have been issued by the counterparty and rank equally with options affect the prices of European options.
\end{abstract}

Keywords. European options, Default risk, Credit spreads, Pricing, Mellin transform.

JEL Classification: G12, G13, C65

\section{Introduction}

In recent years, the over-the-counter (OTC) options markets form a significant proportion of the financial markets. However, unlike the options traded in regulated exchanges, there is no organizing exchange in OTC markets requiring that options positions are marked to market on a daily basis and sufficient collateral posted. Hence, the option holder is always prone to counterparty credit risks because the option writer of the counterparty may not make the promised payments at the exercise date. Thus, the credit risk has to be put forward when pricing the OTC options. To measure credit risk, there are two basic frameworks: structural and reduced-form models. It is well-known that the structural approach for credit default risk proposed by Black \& Scholes (1973) and Merton (1974). The structural approach is intuitive because it links default risk to the firm's economic fundamentals. In contrast to the structural approach, the reduced-form approach models a firm's default time by exogenously specifying a hazard rate or assumes that it is driven by a default intensity that is a function of latent state variables, 
related literatures include those of Hull \& White (1995), Jarrow\& Turnbull (1995), Jarrow et al. (1997), Lee et al. (2017), and Wang (2018). Owing to its

mathematical tractability, the reduced-form approach to modeling credit risk has become very popular among researchers and practitioners.

Based on the default model of corporate bonds proposed by Merton (1974), Johnson \& Stulz (1987)obtained a pricing formula under the assumption that the option holder would receive the total assets of the option writer in default at the expiration date and that the option could be treated as the only liability in the option writer's capital structure. However, the option being the option writer's only obligation in Johnson \& Stulz (1987) model is inconsistent with real-world business situations, Hull \& White (1995)allowed the counterparty to have other liabilities of equal priority, and they adopted the reduced-form approach to estimate the impact of default on bonds issued by the counterparty and ranking equally with the derivative security. In this paper, we follow and extend the work of Hull \& White (1995) to price vulnerable European options, which is not only to allow the option writer to have bond liabilities of equal priority payment under the option but also credit spreads depend only on the price of bonds for reflecting default risk in the calculated price of OTC options is stochastic. In the reduced form framework, the credit events are considered to be governed by exogenous reason stochastically, see Duffie\& Singleton (1999), Jarrow \& Turnbull (1995), and Lando (1998). Longstaff\& Schwartz (1995)pointed out that these dynamics imply that changes in credit spreads are mean-reverting and homoscedastic, which is consistent with the empirical data. Furthermore, these dynamics imply that credit spreads are positive and conditionally lognormally distributed. Therefore, following Longstaff\& Schwartz (1995) and Rizal et al. (2018),we use stochastic model of Vasicek as an exogenous process to model credit risk spreads.

Up to now, to find the analytic formula for the valuation of options, most of the previous literature has used mainly probabilistic techniques. However, the pricing of a given option with probabilistic approaches requires the complexity of the calculation. To solve this problem, the Mellin transform approach has been used to obtain the pricing formulas of the various options, we can refer to we can refer to Gzyl et al. (2017), Jeon et al. (2017), Jeon\& Kim (2019), Kim \& Koo (2016), LI \& Rodrigo (2017), Panini \&Srivastav (2004), and Yoon \& Kim (2015). The Mellin transform is an integral transform, which is regarded as the multiplicative version of the two-sided Laplace transform. In particular, the Mellin transform approach exploits the properties of this transform to reduce the pricing PDE into an ODE that can be solved easily. The option price is then recovered by the convolution property. Therefore, this method will help us to obtain the analytic integral formula of vulnerable European options with stochastic credit spreads more easily and effectively.

The rest of the paper proceeds as follows. Section 2 derives a closed-form formula for vulnerable European option by employing Mellin transform techniques. Numerical examples are shown in Section 3. Section 4 concludes. 


\section{Model formulation}

In this section, we investigate the price of vulnerable European option with stochastic credit spreads. By using Mellin transform, we derive an explicit closedform solution.

\subsection{Modeling assumptions}

As in Hull \&White (1995), we need the following notation:

$P=$ current price of vulnerable European option under consideration,

$P^{*}=$ current price of European option assuming no defaults,

$r=$ the short term risk-free interest rate,

$\gamma=$ the proportional recovery made in the event of default,

$\theta=$ denote vector of state variables determining the $P^{*}$ and $r$ variables,

$\phi=$ denote vector of state variables determining the occurrence defaults and $\gamma$,

Then the current price of vulnerable European put option is written as

$$
P=\hat{E}_{\theta, \phi}\left[\exp \left(-\int_{t}^{T} r d s\right) \gamma_{b} P_{T}^{*}\right]
$$

where the expectations operator $E$ is taken over all paths for $\theta$ and $\phi$ between times $t$ and $T, b$ denote the first payoff boundary point crossed by the path. Since $r$ is defined by $\theta$ variables, equation (1) can be written

$$
P=\hat{E}_{\theta}\left[\exp \left(-\int_{t}^{T} r d s\right) \hat{E}_{\phi \mid \theta}\left(\gamma_{b} P_{T}^{*}\right)\right] .
$$

Defining

$$
\omega(\theta, T)=\hat{E}_{\phi \mid \theta}\left(\gamma_{b}\right)
$$

Suppose the state variables comprising $\theta$ are independent of the state variables comprising $\phi$, then equation (3) becomes

$$
\omega(\theta, T)=\hat{E}_{\theta}\left(\gamma_{b}\right)
$$

The function $\omega(\theta, T)$ is independent of $\theta$ can be written as $\omega(T)$, Equation (2) becomes

$$
P=\omega(T) \hat{E}_{\theta}\left[\exp \left(-\int_{t}^{T} r d s\right) P_{T}^{*}\right] .
$$

When there is no possibility of defaults, then the price of vulnerable European option can be written

$$
P^{*}=\hat{E}_{\theta}\left[\exp \left(-\int_{t}^{T} r d s\right) P_{T}^{*}\right]
$$

with payoff function $g$ is given by

$$
g\left(S_{T}\right)=\left(K-S_{T}\right)^{+} \text {. }
$$


Let $B$ and $B^{*}$ denote current value of a vulnerable zero-coupon bond issued by the option writer that pays off $\$ 1$ at time $t$ to maturity $T$ and ranks equally with the option in the event of a default and default-free zero-coupon bond, respectively. Then, similarly for the value of zero-coupon bond is given

$$
B=\omega(T) \hat{E}_{\theta}\left[\exp \left(-\int_{t}^{T} r d s\right)\right] .
$$

Also

$$
B^{*}=\hat{E}_{\theta}\left[\exp \left(-\int_{t}^{T} r d s\right)\right] .
$$

From (5), (6), (8) and (9), we get

$$
P=\omega(T) P^{*} \frac{B}{B^{*}} P^{*} .
$$

Defining $y$ and $y^{*}$ (equals $r$ ) as the yields on $B$ and $B^{*}$, respectively. Because $y=\frac{-\log B}{T-t}$, then equation (10) becomes

$$
P=\exp \left(-\left(y-y^{*}\right)(T-t)\right) P^{*}
$$

Then, the no-arbitrage price of a vulnerable European put option under the martingale measure is written by

$$
P=\hat{E}_{\theta}\left[\exp \left(-\left(y-y^{*}\right)(T-t)\right) g\left(S_{T}\right)\right],
$$

where $y^{\prime}=y-y^{*}$ represents credit spreads between $B$ and $B^{*}, S$ is current price of the stock, $K$ is the strike price of the option. Then equation (12) is considered as a discount function for $g\left(S_{T}\right)$.

We suppose that the risk-neutral process for $S$ and $y^{\prime}$ satisfied following stochastic differential equations (in short, SDE)

$$
\begin{aligned}
d S & =r S d t+\sigma_{s} S d W_{s}, \\
d y^{\prime} & =\kappa\left(\alpha-y^{\prime}\right) d t+\sigma_{y} d W_{y^{\prime}},
\end{aligned}
$$

where $y$ is the credit spread rate, $\alpha$ is the mean credit spread rate in long term, $\kappa$ is the coefficient of the reversion speed of credit spread rate towards its long-run mean $\alpha, \sigma_{s}$ is the volatility of the underlying asset, $\sigma_{y}$ is the credit spread volatility. The Wiener process $d W_{s}$ and $d W_{y^{\prime}}$ are independent with $d W_{s} d W_{y^{\prime}}=0$.

The price of vulnerable European option is $P\left(t, S, y^{\prime}, T\right)$. By applying Feynman-Kac theorem, the partial differential equation (PDE) governing the option value is 


$$
\frac{\partial P}{\partial t}+\frac{1}{2} \sigma_{s}^{2} S \frac{\partial^{2} P}{\partial t^{2}}+\frac{1}{2} \sigma_{y}^{2} \frac{\partial^{2} P}{\partial y^{\prime 2}}+r S \frac{\partial P}{\partial S}+\kappa\left(\alpha-y^{\prime}\right) \frac{\partial P}{\partial y^{\prime}}=\left(y^{\prime}+r\right) P,
$$

with the terminal condition $P\left(t, S, y^{\prime}, T\right)=g\left(S_{T}\right)$.

\subsection{Vulnerable option price formula}

The objective of this subsection is to derive a vulnerable European option pricing formula by solving (15) through the Mellin transform method. Therefore, we first summarize the definition and some basic properties of without proof for readers who are unfamiliar with the Mellin transforms. The interested reader can refer to Erdelyi et al.(1954) and Sneddon(1972) as well.

Definition 2.1(The Mellin transform and inverse Mellin transform). The Mellin transform $\hat{g}(s)$ of a complex-valued function $g(x)$ defined over positive reals is

$$
M_{x}[g(x) ; s]:=\hat{g}(s)=\int_{0}^{\infty} g(x) x^{-1} d x,
$$

with $s$ being a complex number. Then the function $g(x)$ can be recovered from its Mellin transform by the inverse Mellin transformation formula

$$
g(x)=M_{x}^{-1}[\hat{g}(s)]=\frac{1}{2 \pi i} \int_{c-i \infty}^{c+i \infty} \hat{g}(s) x^{-s} d s,
$$

where $c$ is any real number.

Proposition 2.1(Convolution of the Mellin transform) Let $f(x)$ and $g(x)$ be locally integrable functions on positive reals. $\hat{f}(s)$ and $\hat{g}(s)$ are two Mellin transforms of the functions $f(x)$ and $g(x)$, respectively. Then, the Mellin convolution is given by the inverse Mellin transform of $\hat{f}(s) \hat{g}(s)$ as follows:

$$
\begin{aligned}
f(x) \vee g(x):= & \frac{1}{2 \pi i} \int_{c-i \infty}^{c+i \infty} \hat{f}(s) \hat{g}(s) x^{-s} d s \\
& =\int_{0}^{\infty} f(\xi) g\left(\frac{x}{\xi}\right) \frac{1}{\xi} d \xi .
\end{aligned}
$$

Proposition 2.2 (Inverse Mellin transform of exponential function) Given complex numbers $\alpha$ and $\beta$ with

$$
\operatorname{Re}(\alpha) \geq 0, \text { let } f(x)=\frac{1}{2 \pi i} \int_{c-i \infty}^{c+i \infty} \hat{f}(s) x^{-s} d s, \text { where } \hat{f}(s)=e^{\alpha(s+\beta)^{2}}
$$

Then holds.

$$
f(x)=\frac{1}{2 \sqrt{\pi \alpha}} x^{\beta} e^{-\frac{(\ln x)^{2}}{4 \alpha}}
$$


Proposition 2.3. (Basic properties of the Mellin transform). Suppose that there exists a Mellin transform of $f(x)$. Then the following relations hold:

$$
\begin{aligned}
& M_{x}\left[x \frac{\partial}{\partial x} f(x) ; s\right]:=-s \hat{f}(s), \\
& M_{x}\left[x^{2} \frac{\partial^{2}}{\partial x^{2}} f(x) ; s\right]:=s(s+1) \hat{f}(s) .
\end{aligned}
$$

Then, by using Proposition 2.3, the Mellin transform of equation (15) yields

$$
-\frac{\partial \hat{P}}{\partial \tau}+\left[\frac{1}{2} \sigma_{s}^{2} \omega^{2}-\left(r-\frac{1}{2} \sigma_{s}^{2}\right) \omega-r-y^{\prime}\right] \hat{P}+\frac{1}{2} \sigma_{y^{\prime}}^{2} \frac{\partial^{2} \hat{P}}{\partial y^{\prime 2}}+\kappa\left(\alpha-y^{\prime}\right) \frac{\partial \hat{P}}{\partial y^{\prime}}=0,
$$

with $\tau=T-t, \hat{P}\left(0, \omega, y^{\prime}\right)=\hat{g}(\omega)$ which is the Mellin transform of $g(S)$

To simplify (23), we let

$$
\hat{P}\left(\tau, \omega, y^{\prime}\right)=\exp \left\{\frac{1}{2} \sigma_{s}^{2} \omega(\omega+1) \tau\right\} \hat{f}\left(\tau, \omega, y^{\prime}\right),
$$

then equation (23) is transformed into the following PDE for $\hat{f}$

$$
-\frac{\partial \hat{f}}{\partial \tau}-\left[(1+\omega) r+y^{\prime}\right] \hat{f}+\frac{1}{2} \sigma_{y^{\prime}}^{2} \frac{\partial^{2} \hat{f}}{\partial y^{\prime 2}}+\kappa\left(\alpha-y^{\prime}\right) \frac{\partial \hat{f}}{\partial y^{\prime}}=0,
$$

with $\hat{f}\left(0, \omega, y^{\prime}\right)=\hat{g}(\omega)$.

To solve (25), we let $\hat{f}\left(\tau, \omega, y^{\prime}\right)=\hat{g}(\omega) G(\tau, \omega) e^{-\left((1+\omega) r+y^{\prime}\right) F(\tau)}$ with terminal condition $\hat{f}\left(0, \omega, y^{\prime}\right)=\hat{g}(\omega)$. By substituting this function form into (25), we get the following ODEs with respect to $G(\tau, \omega)$ and $F(\tau)$

$$
\left\{\begin{array}{c}
\frac{\partial G}{\partial \tau}-\frac{1}{2} \sigma_{y}^{2} F^{2} G+\kappa(\alpha+(1+\omega) r) F G=0 \\
\frac{\partial F}{\partial \tau}+\kappa F-1=0
\end{array}\right.
$$

where $G(0, \omega)=1$ and $F(0)=0$. By solving ODEs (26), we have

$$
\left\{\begin{array}{c}
G(\tau, \omega)=\exp \left(\frac{\sigma_{y}^{2}}{2 \kappa^{2}}\left(\tau-F(\tau)-\frac{\kappa}{2} F^{2}(\tau)\right)-(\alpha+(1+\omega) r)(\tau-F(\tau))\right), \\
F(\tau)=\frac{1-e^{-\kappa \tau}}{\kappa},
\end{array}\right.
$$

Then, by substituting $G(\tau, \omega), F(\tau)$ and $\hat{f}\left(\tau, \omega, y^{\prime}\right)$ into (24), we have 


$$
\hat{P}\left(\tau, \omega, y^{\prime}\right)=\hat{g}(\omega) \exp \left\{\hat{Q}\left(\tau, \omega, y^{\prime}\right)\right\},
$$

where

$$
\begin{gathered}
\hat{Q}\left(\tau, \omega, y^{\prime}\right)=D(\tau)+\frac{1}{2} \sigma_{s}^{2} \omega(\omega+1) \tau-(1+\omega) r \tau-\alpha(\tau-F(\tau))-y^{\prime} F(\tau), \\
D(\tau)=\frac{\sigma_{y^{\prime}}^{2}}{2 \kappa^{2}}\left(\tau-F(\tau)-\frac{\kappa}{2} F^{2}(\tau)\right) .
\end{gathered}
$$

Hence, the price of vulnerable European put option is given

$$
P\left(\tau, S, y^{\prime}\right)=\frac{1}{2 \pi i} \int_{c-i \infty}^{c+i \infty} \hat{g}(\omega) \exp \left\{\hat{Q}\left(\tau, \omega, y^{\prime}\right)\right\} S^{-\omega} d \omega
$$

Let

$$
\begin{aligned}
\varphi\left(\tau, S, y^{\prime}\right) & =\frac{1}{2 \pi i} \int_{c-i \infty}^{c+i \infty} \exp \left\{\hat{Q}\left(\tau, \omega, y^{\prime}\right)\right\} S^{-\omega} d \omega \\
& =e^{M^{*}(\tau)} \frac{1}{2 \pi i} \int_{c-i \infty}^{c+i \infty} \exp \left\{E(\tau)\left(\omega+\frac{E(\tau)+M(\tau)}{2 E(\tau)}\right)^{2}\right\} S^{-\omega} d \omega,
\end{aligned}
$$

where

$$
\begin{gathered}
E(\tau)=\frac{1}{2} \sigma_{s}^{2} \tau \\
M(\tau)=-r \tau \\
M^{*}(\tau)=D(\tau)-\alpha(\tau-F(\tau))-y^{\prime} F(\tau)-r \tau-\frac{(E(\tau)+M(\tau))^{2}}{4 E(\tau)} .
\end{gathered}
$$

Because $E(\tau)>0$ holds for $\tau>0$, by using Proposition 2.2, we obtain

$$
\varphi\left(\tau, S, y^{\prime}\right)=\frac{1}{2 \sqrt{\pi E(\tau)}} S^{-\frac{E(\tau)+M(\tau)}{2 E(\tau)}} \exp \left\{M^{*}(\tau)-\frac{(\ln S)^{2}}{4 E(\tau)}\right\},
$$

Since $\hat{g}(\omega)$ and $\exp \left\{\hat{Q}\left(\tau, \omega, y^{\prime}\right)\right\}$ are the Mellin transform of $g(S)$ and $\varphi\left(\tau, S, y^{\prime}\right)$, respectively. Using Proposition 2.1, we have the following result

$$
\begin{aligned}
P\left(\tau, S, y^{\prime}\right) & =\int_{0}^{\infty} g(u) \varphi\left(\frac{S}{u}, y^{\prime}\right) u^{-1} d u \\
& =\int_{0}^{K}(K-u) \varphi\left(\frac{S}{u}, y^{\prime}\right) u^{-1} d u
\end{aligned}
$$

Setting 


$$
\begin{gathered}
\theta_{1}(u)=\frac{1}{2 \sqrt{\pi E(\tau)}}\left(\frac{S}{u}\right)^{-\frac{E(\tau)+M(\tau)}{2 E(\tau)}}, \\
\theta_{2}(u)=M^{*}(\tau)-\frac{(\ln S)^{2}}{4 E(\tau)} .
\end{gathered}
$$

Then equation (30) yields

$$
P\left(\tau, S, y^{\prime}\right)=\int_{0}^{K} \theta_{1}(u)(K-u) \exp \left(\theta_{2}(u)\right) u^{-1} d u .
$$

Now we give a closed-form formula for vulnerable European put option price.

Theorem 2.1For the value of a vulnerable European put option, defined by (12) can be expressed as the following pricing formula:

$$
P\left(\tau, S, y^{\prime}\right)=R\left(y^{\prime}, \tau\right)\left[e^{-r \tau} K N\left(-d_{2}\right)-S N\left(-d_{1}\right)\right],
$$

where $N$ is the standard normal cumulative distribution as

$$
N(x)=\frac{1}{\sqrt{2 \pi}} \int_{-\infty}^{x} e^{-\frac{1}{2} s^{2}} d s,
$$

and

$$
\begin{gathered}
R\left(y^{\prime}, \tau\right)=A(\tau) \exp \left(-y^{\prime} F(\tau)\right), \\
A(\tau)=\exp \left(\left(\alpha-\frac{\sigma_{y^{\prime}}^{2}}{2 \kappa^{2}}\right)(F(\tau)-\tau)-\frac{\sigma_{y^{\prime}}^{2}}{4 \kappa} F^{2}(\tau)\right), \\
F(\tau)=\frac{1-e^{-\kappa \tau}}{\kappa} \\
d_{1}=\frac{\ln \left(\frac{S}{K}\right)+\left(\frac{1}{2} \sigma_{s}^{2}+r\right) \tau}{\sigma_{s} \sqrt{\tau}} \\
d_{2}=d_{1}-\sigma_{s} \sqrt{\tau}
\end{gathered}
$$

Proof. First of all, let $x=\frac{\ln \left(\frac{S}{u}\right)}{\sqrt{2 E(\tau)}}$. By applying the change of variable from $u$ to $x$, we then get 


$$
\begin{aligned}
P\left(\tau, S, y^{\prime}\right)= & -\frac{1}{\sqrt{2 \pi}} \int_{\infty}^{\ln \left(\frac{S}{K}\right)} \frac{\sqrt{2 E(\tau)}}{\sqrt{2 E}}\left(K-S e^{-\sqrt{2 E(\tau)} x}\right) \exp \left(M^{*}(\tau)-\frac{1}{2} x^{2}\right) d x \\
= & -\frac{K}{\sqrt{2 \pi}} \int_{\infty}^{\ln \left(\frac{S}{K}\right)} \frac{\sqrt{2 E(\tau)}}{\ln } \exp \left(-\frac{1}{2} x^{2}+\frac{E(\tau)+M(\tau)}{2 E(\tau)} x+M^{*}(\tau)\right) d x \\
& +\frac{S}{\sqrt{2 \pi}} \int_{\infty}^{\ln \left(\frac{S}{K}\right)} \frac{\sqrt{2 E(\tau)}}{\exp }\left(-\frac{1}{2} x^{2}+\frac{-E(\tau)+M(\tau)}{2 E(\tau)} x+M^{*}(\tau)\right) d x .
\end{aligned}
$$

To evaluate the first term in (34), we introduce an auxiliary function

$$
I_{1}=-\frac{K}{\sqrt{2 \pi}} \int_{\infty}^{\frac{\ln \left(\frac{S}{K}\right)}{\sqrt{2 E(\tau)}}} \exp \left(-\frac{1}{2} x^{2}+\frac{E(\tau)+M(\tau)}{2 E(\tau)} x+M^{*}(\tau)\right) d x
$$

Then the exponent of the integrand of (35) can be expressed as

$$
M_{2}-\frac{1}{2}(x+b)^{2}
$$

where $b$ and $M_{2}$ are given by

$$
\begin{gathered}
b=-\frac{E(\tau)+M(\tau)}{\sqrt{2 E(\tau)}}=-\frac{\left(\frac{1}{2} \sigma_{s}^{2}-r\right) \tau}{\sigma_{s} \sqrt{\tau}}, \\
M_{2}=M^{*}(\tau)+\frac{b^{2}}{2}=\left(\alpha-\frac{\sigma_{y^{\prime}}^{2}}{2 \kappa^{2}}\right)(F(\tau)-\tau)-\frac{\sigma_{y^{\prime}}^{2}}{4 \kappa} F^{2}(\tau)-y^{\prime} F(\tau)-r \tau,
\end{gathered}
$$

Therefore

$$
R\left(y^{\prime}, \tau\right)=A(\tau) \exp \left(-y^{\prime} F(\tau)\right)
$$

where

$$
\begin{gathered}
A(\tau)=\exp \left(\left(\alpha-\frac{\sigma_{y^{\prime}}^{2}}{2 \kappa^{2}}\right)(F(\tau)-\tau)-\frac{\sigma_{y^{\prime}}^{2}}{4 \kappa} F^{2}(\tau)\right), \\
F(\tau)=\frac{1-e^{-\kappa \tau}}{\kappa} .
\end{gathered}
$$
function

Similarly, to evaluate the second term in (34), we introduce an auxiliary 


$$
I_{2}=-\frac{S}{\sqrt{2 \pi}} \int_{\infty}^{\frac{\ln \left(\frac{S}{K}\right)}{\sqrt{2 E(\tau)}}} \exp \left(-\frac{1}{2} x^{2}+\frac{-E(\tau)+M(\tau)}{2 E(\tau)} x+M^{*}(\tau)\right) d x
$$

Then the exponent of the integrand of (36) can be expressed as

$$
M_{1}-\frac{1}{2}(x+a)^{2}
$$

where $a$ and $M_{1}$ are given by

$$
\begin{gathered}
a=-\frac{-E(\tau)+M(\tau)}{\sqrt{2 E(\tau)}}=\frac{\left(\frac{1}{2} \sigma_{s}^{2}+r\right) \tau}{\sigma_{s} \sqrt{\tau}}, \\
M_{1}=M^{*}(\tau)+\frac{a^{2}}{2}=R\left(y^{\prime}, \tau\right) .
\end{gathered}
$$

Therefore, using change of variables

$$
\begin{aligned}
& x_{1}=x+\frac{\left(\frac{1}{2} \sigma_{s}^{2}+r\right) \tau}{\sigma_{s} \sqrt{\tau}}, \\
& x_{2}=x-\frac{\left(\frac{1}{2} \sigma_{s}^{2}-r\right) \tau}{\sigma_{s} \sqrt{\tau}} .
\end{aligned}
$$

Then, $P\left(\tau, S, y^{\prime}\right) \mathrm{P}$ is given by the formula

$$
\begin{aligned}
P\left(\tau, S, y^{\prime}\right) & =-\frac{K R\left(y^{\prime}, \tau\right) e^{-r \tau}}{\sqrt{2 \pi}} \int_{\infty}^{d_{2}} \exp \left(-\frac{1}{2} x_{2}^{2}\right) d x_{2}+\frac{S R\left(y^{\prime}, \tau\right)}{\sqrt{2 \pi}} \int_{\infty}^{d_{1}} \exp \left(-\frac{1}{2} x_{1}^{2}\right) d x_{1} \\
& =R\left(y^{\prime}, \tau\right)\left[e^{-r \tau} K N\left(-d_{2}\right)-S N\left(-d_{1}\right)\right],
\end{aligned}
$$

where

$$
\begin{aligned}
& d_{1}=\frac{\ln \left(\frac{S}{K}\right)+\left(\frac{1}{2} \sigma_{s}^{2}+r\right) \tau}{\sigma_{s} \sqrt{\tau}}, \\
& d_{2}=d_{1}-\sigma_{s} \sqrt{\tau} \text {. }
\end{aligned}
$$

Remark 2.1If there is no credit risk(i.e., $R\left(y^{\prime}, \tau\right)=1$ ), that is, $B=B^{*}=e^{-r \tau}$, then (33) reduces to

$$
P\left(\tau, S, y^{\prime}\right)=e^{-r \tau} K N\left(-d_{2}\right)-S N\left(-d_{1}\right)
$$

where 


$$
\begin{gathered}
d_{1}=\frac{\ln \left(\frac{S}{K}\right)+\left(\frac{1}{2} \sigma_{s}^{2}+r\right) \tau}{\sigma_{s} \sqrt{\tau}}, \\
d_{2}=d_{1}-\sigma_{s} \sqrt{\tau},
\end{gathered}
$$

which is exactly the Black-Scholes's pricing formula for European put options.

Remark 2.2 If we assume that credit risk is measured by constant credit spread (i.e., $R\left(y^{\prime}, \tau\right)=e^{-\left(y^{\prime}+r\right) \tau}$ ), then, (33) becomes the pricing formula in Hull \& White (1995)

$$
\begin{gathered}
P\left(\tau, S, y^{\prime}\right)=e^{-\left(y^{\prime}+r\right) \tau} K N\left(-d_{2}\right)-e^{-y^{\prime} \tau} S N\left(-d_{1}\right), \\
d_{1}=\frac{\ln \left(\frac{S}{K}\right)+\left(\frac{1}{2} \sigma_{s}^{2}+r\right) \tau}{\sigma_{s} \sqrt{\tau}}, \\
d_{2}=d_{1}-\sigma_{s} \sqrt{\tau} .
\end{gathered}
$$

The proposed model formula (33) is also in the form of Hull \& White (1995) (similar to the Black-Scholes type options formula). However, its component is different, because the credit risk depends only on the prices of bonds that have been issued by the counterparty and rank equally with European options is expressed by $R\left(y^{\prime}, \tau\right)=A(\tau) \exp \left(-y^{\prime} F(\tau)\right)$. But, in the Hull and White model, the credit risk is measured by $e^{-\left(y-y^{*}\right) \tau}$.

Theorem 2.2 For vulnerable European call option pricing formula is given by

$$
C\left(\tau, S, y^{\prime}\right)=R\left(y^{\prime}, \tau\right)\left[S N\left(d_{1}\right)-e^{-r \tau} K N\left(d_{2}\right)\right],
$$

where $d_{1}, d_{2}$ and $R\left(y^{\prime}, \tau\right)$ are defined by the theorem 2.1 .

\section{Numerical Experiments}

In this section, we give the sensitivity analysis in order to explore the impact of different parameters on option prices, when the credit risk depends only on the prices of bonds that have been issued by the counterparty and rank equally with options. Given a vulnerable European put option, we use the following value of the parameters whenever they are required to be specified throughout the numerical analysis unless indicated otherwise: $S_{0}=K=1, \tau=1, y_{0}^{\prime}=0.04, r=0.05$, $\sigma_{s}=0.25, \sigma_{y}=0.15, \kappa=0.12, \alpha=0.08$.

Table 1 compares the option prices given by our proposed model to those in Black \& Scholes (1973) and Hull \& White (1995) for various choices of the underlying asset volatility, the credit spread volatility, speed of adjustment, and 
long-run mean. The options pries in the proposed model $(\mathrm{P})$, the Black and Scholes model (BS) and the Hull and White model (HW) are computed by (33), (38) and (39), respectively. For further comparison of $\mathrm{P}$ with BS and HW, we compute the percentage difference $\Delta \% \equiv 100(P-B S) / B S$ or $\Delta \% \equiv 100(P-H W) / H W$. Four points are noteworthy from Table 1. First, the option prices in the BS model are higher than those in the proposed model and the HW model due to the default risk from the counterparty. Second, increasing the underlying asset volatility increases the value of European put options, no matter which model is applied. This is because a higher volatility of the underlying asset causing a higher premium of the option. In addition, we can understand the effect of $\sigma_{y}$ in the same way. Such a phenomenon is observed clearly in Figure 2 and figure 3.Third, when the long-run mean $(\alpha)$ is close to the initial level of credit spread ( $y_{0}^{\prime}=0.04$ ), our proposed model and the Hull and White model prices are not significantly different. As $\alpha$ deviates from the initial credit spread value, the difference in prices between the proposed model and the Hull and White model increases. On the one hand, if $\alpha$ is higher than $y_{0}^{\prime}=0.04$, then the Hull and White model drastically overprices the option. This is because, with use of the Hull and White model, credit spread is assumed to remain constant at its initial value. Since the option price is decreasing function of the credit spread rate, keeping the credit spread rate at a lower level leads to a higher option price. However, in the proposed model, the credit spread rate increases towards $\alpha$ during the life of the contract and the option price is lower than when credit spread had been kept at the lower initial level. On the other hand, if $\alpha$ is lower than $y_{0}^{\prime}=0.04$, the opposite conclusion is reached. Fourth, as the speed of adjustment $(\kappa)$ increases, the difference in prices between the proposed model and the Hull and White model increases. This is because with a high speed of adjustment $(\kappa)$, credit spread returns to its long-run level faster, but in the Hull and White model, credit spread is kept at a higher level through the entire life of the option contract. Therefore, the higher $\kappa$, the higher is our proposed model price and the greater is difference in prices between our proposed model and the Hull and White model. 
Closed Form Valuation of Vulnerable European Options with Stochastic Credit Spreads

\begin{tabular}{|c|c|c|c|c|c|c|c|c|c|}
\hline & & Parameter & Values & & Ontion & & Dris & \multicolumn{2}{|c|}{$\mathrm{Va}$} \\
\hline & & & & & & & & $\begin{array}{c}\text { Difference } \\
\text { Between BS and } \\
\text { P }\end{array}$ & $\begin{array}{c}\text { Difference } \\
\text { Between BS and } \\
\text { P }\end{array}$ \\
\hline & $\sigma_{s}$ & $\sigma_{y^{\prime}}$ & $\kappa$ & $\alpha$ & BS & HW & $\mathrm{P}$ & $\Delta \% \equiv 100 \Delta / B S$ & $\Delta \% \equiv 100 \Delta / H W$ \\
\hline In-the & 0.25 & 0.15 & 0.12 & 0.02 & 0.1826 & 0.1755 & 0.1299 & -28.8700 & -25.9671 \\
\hline $\begin{array}{l}\text { money } \\
\text { options }\end{array}$ & 0.30 & 0.15 & 0.12 & 0.02 & 0.1968 & 0.1890 & 0.1400 & -28.8700 & -25.9671 \\
\hline \multirow[t]{10}{*}{$S_{0}=0.8$} & 0.50 & 0.15 & 0.12 & 0.02 & 0.2585 & 0.2484 & 0.1839 & -28.8700 & -25.9671 \\
\hline & 0.25 & 0.20 & 0.12 & 0.02 & 0.1826 & 0.1755 & 0.1027 & -43.7523 & -41.4568 \\
\hline & 0.25 & 0.25 & 0.12 & 0.02 & 0.1826 & 0.1755 & 0.0760 & -58.4062 & -56.7088 \\
\hline & 0.25 & 0.25 & 1.15 & 0.02 & 0.1826 & 0.1755 & 0.1779 & -2.61210 & 1.3624 \\
\hline & 0.25 & 0.25 & 3.20 & 0.02 & 0.1826 & 0.1755 & 0.1783 & -2.37100 & 1.6133 \\
\hline & 0.25 & 0.25 & 3.20 & 0.03 & 0.1826 & 0.1755 & 0.1771 & -3.0522 & 0.9043 \\
\hline & 0.25 & 0.25 & 3.20 & 0.04 & 0.1826 & 0.1755 & 0.1758 & -3.7287 & 0.2002 \\
\hline & 0.25 & 0.25 & 3.20 & 0.05 & 0.1826 & 0.1755 & 0.1746 & -4.4005 & -0.4990 \\
\hline & 0.25 & 0.25 & 3.20 & 0.08 & 0.1826 & 0.1755 & 0.1710 & -6.38780 & -2.5674 \\
\hline & 0.25 & 0.25 & 3.20 & 0.10 & 0.1826 & 0.1755 & 0.1686 & -7.6897 & -3.9224 \\
\hline
\end{tabular}

DOI: $10.24818 / 18423264 / 53.4 .19 .18$ 
Zong-Gang Ma, Shi-Song Xiao

\begin{tabular}{|c|c|c|c|c|c|c|c|ccc|}
\hline At-the- & 0.25 & 0.15 & 0.12 & 0.01 & 0.0746 & 0.0717 & 0.0531 & -28.8290 & -25.9244 \\
\hline $\begin{array}{c}\text { money } \\
\text { options }\end{array}$ & 0.15 & 0.15 & 0.12 & 0.01 & 0.0371 & 0.0357 & 0.0264 & -28.8290 & -25.9244 \\
\hline$S_{0}=1$ & 0.10 & 0.15 & 0.12 & 0.01 & 0.0193 & 0.0185 & 0.0137 & -28.8290 & -25.9244 \\
& 0.10 & 0.20 & 0.12 & 0.01 & 0.0193 & 0.0185 & 0.0109 & -43.7199 & -41.4230 \\
& 0.10 & 0.30 & 0.12 & 0.01 & 0.0193 & 0.0185 & 0.0055 & -71.2211 & -70.0466 \\
& 0.10 & 0.30 & 2.20 & 0.01 & 0.0193 & 0.0185 & 0.0189 & -1.7196 & 2.2913 \\
& 0.10 & 0.30 & 3.30 & 0.01 & 0.0193 & 0.0185 & 0.0190 & -1.5878 & 2.4285 \\
& 0.10 & 0.30 & 3.30 & 0.03 & 0.0193 & 0.0185 & 0.0187 & -2.9718 & 0.9880 \\
& 0.10 & 0.30 & 3.30 & 0.04 & 0.0193 & 0.0185 & 0.0186 & -3.6565 & 0.2754 \\
& 0.10 & 0.30 & 3.30 & 0.05 & 0.0193 & 0.0185 & 0.0184 & -4.3363 & -0.4322 \\
Out-of-the & 0.10 & 0.30 & 3.30 & 0.08 & 0.0193 & 0.0185 & 0.0181 & -6.3472 & -2.5251 \\
money & 0.10 & 0.30 & 3.30 & 0.10 & 0.0193 & 0.0185 & 0.0178 & -7.6642 & -3.8959 \\
options & 0.25 & 0.15 & 2.20 & 0.01 & 0.0129 & 0.0124 & 0.0127 & -2.0713 & 1.9253 \\
$S_{0}=1.2$ & 0.30 & 0.15 & 2.20 & 0.01 & 0.0253 & 0.0243 & 0.0248 & -2.0713 & 1.9253 \\
& 0.30 & 0.50 & 2.20 & 0.01 & 0.0400 & 0.0385 & 0.0397 & -0.8808 & 3.1643
\end{tabular}


Closed Form Valuation of Vulnerable European Options with Stochastic Credit Spreads

\begin{tabular}{llllllllllll}
\hline 0.30 & 0.60 & 2.20 & 0.01 & 0.0400 & 0.0385 & 0.0399 & -0.3000 & 3.7688 \\
0.20 & 0.15 & 1.15 & 0.01 & 0.0129 & 0.0124 & 0.0126 & -2.5545 & 1.4223 \\
0.20 & 0.15 & 0.12 & 0.01 & 0.0129 & 0.0124 & 0.0092 & -28.8290 & -25.9244 \\
0.20 & 0.15 & 2.20 & 0.03 & 0.0129 & 0.0124 & 0.0125 & -3.2313 & 0.7179 \\
0.20 & 0.15 & 2.20 & 0.04 & 0.0129 & 0.0124 & 0.0124 & -3.8062 & -0.1196 \\
0.20 & 0.15 & 2.20 & 0.05 & 0.0129 & 0.0124 & 0.0124 & -4.3776 & -0.4752 \\
0.20 & 0.15 & 2.20 & 0.08 & 0.0129 & 0.0124 & 0.0121 & -6.0716 & -2.2383 \\
0.20 & 0.15 & 2.20 & 0.10 & 0.0129 & 0.0124 & 0.0120 & -7.1843 & -3.3964
\end{tabular}

Note: $\mathrm{BS}=$ the Black and Scholes price, $\mathrm{HW}=$ the Hull and White price, and $\mathrm{P}=$ the proposed model price from (33) 


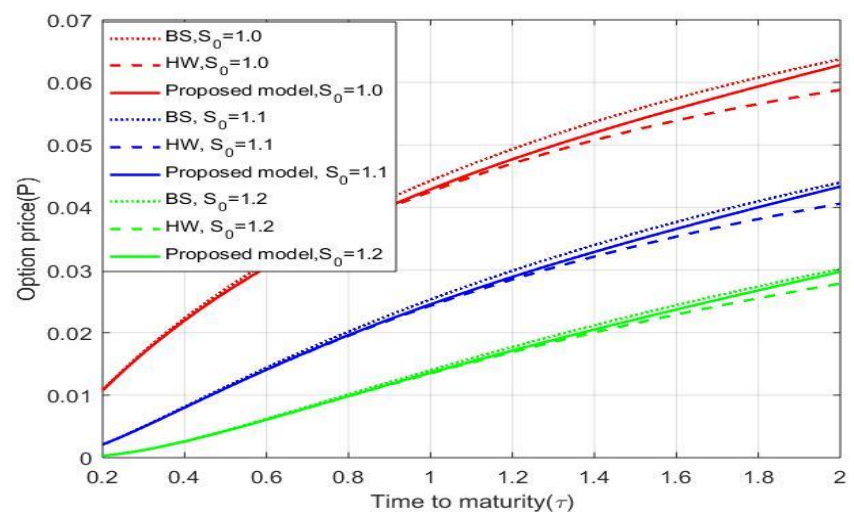

Figure 1: European put option prices with different $S_{0}$ varying $\tau$.

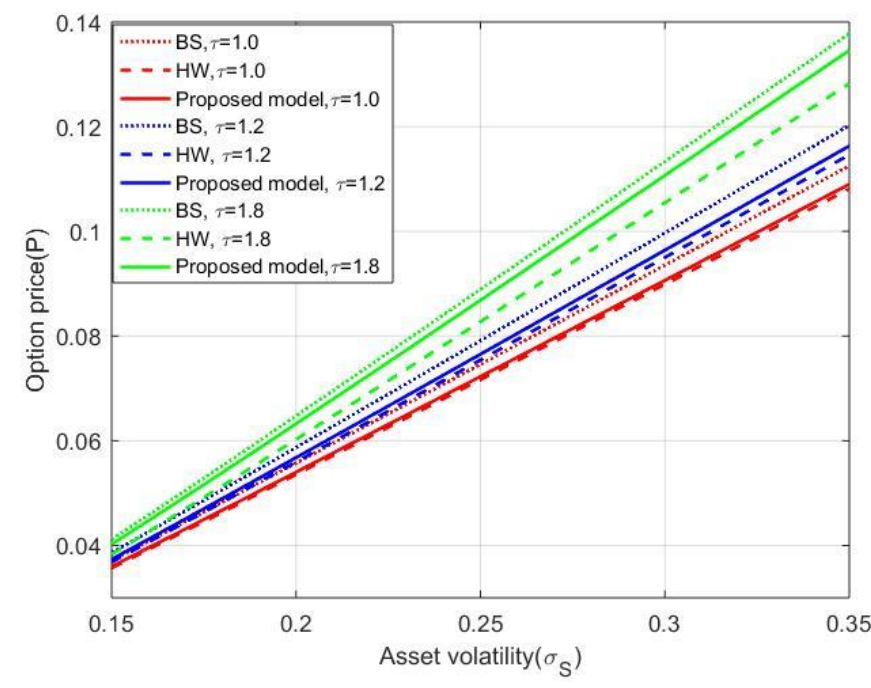

Figure 2: European put option prices with different time to maturity $\tau$ varying $\sigma_{s}$

Figure 1 plots European put option prices against time to maturity $\tau$ for three different scenarios namely $S_{0}=1, S_{0}=1.1$ and $S_{0}=1.2$. One can observe that European put option prices increase with an increase in the time to maturity no matter what the asset price is chosen. This agrees with the fact that option prices are increasing function of time to maturity. Also, the option prices are higher when there is no risk of default by the counterparty as compared to the cases with the risk of default. From Figure 1, we also can observe that a higher underlying asset price leads to lower European put option price. Furthermore, we analyze the sensitivity of vulnerable European option prices with respect to changes in the parameters that 
come into play due to the volatility of underlying asset and credit spread. Figure 2 and Figure 3 illustrate the sensitivity of European put option with respect to the volatility of the underlying asset and the volatility of credit spread for different time to maturity, $\tau=1.0,1.2,1.8$. Figure 2 shows that with the high values of the underlying asset price volatility $\sigma_{s}$ can lead to a higher vulnerable price. Figure 3 displays the same case with respect to the credit spread volatility in the proposed model. We can observe that the high credit spread volatility can lead to a higher option price. Figure 4 demonstrates that the option prices decrease as the initial credit spread value $y_{0}^{\prime}$ increases.

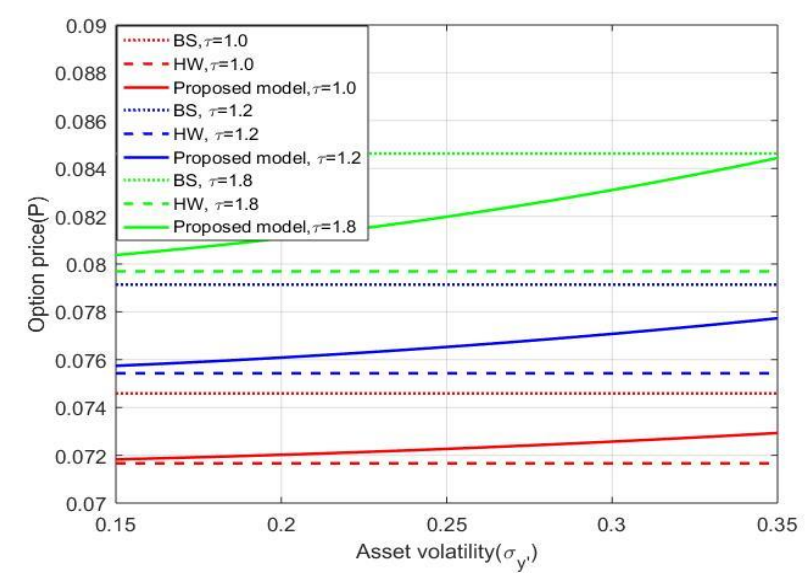

Figure 3:European put prices with different time to maturity $\tau$ varying $\sigma_{y}$.

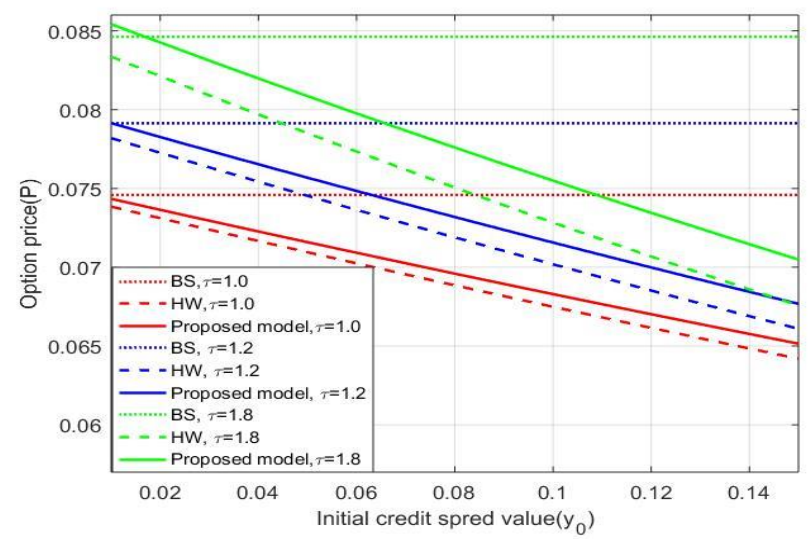

Figure 4:European put prices with different time to maturity $\tau$ varying $y_{0}$.

\section{Conclusions}

This paper extends the vulnerable European option pricing results of to allow not only for other liabilities in the capital structure of the option writer but also for credit spread is stochastic. Specially, we derive the closed-formed pricing formulas 
of vulnerable European options by applying the Mellin transforms techniques. We also provided the table and graphs to illustrate the significant movements of the prices with respect to parameters of option. The analysis here suggests that a constant credit spread model can lead to significantly different options prices relative to those obtained by a stochastic credit spread. Variables important in determine vulnerable options prices are the initial level of credit spread, the speed of adjustment of credit spread, the long-run mean rate, and the time to maturity. There does not appear to be a significant difference between the proposed model and the HW model when the initial credit spread rate is close to long-run mean rate $\alpha$ ). In addition, the proposed formula predicts lower option prices than those predicted by the Black and Scholes formula for in-the-money, at-the-money options and out-of-the-money. However, the proposed formula predicts higher option prices than those predicted by the Hull and White formula when the initial credit spread rate is less than long-run mean rate, otherwise, the opposite conclusion is reached. In conclude, our results suggest that the incorporation of a stochastic credit spread is extremely important when valuing vulnerable European options, particularly when the credit risk depends only on the prices of bonds that have been issued by the counterparty and rank equally with European options.

\section{ACKNOWLEDGMENTS}

This research was supported by the China Scholarship Council (201608440451), the social science and humanity on Young Fund of the Ministry of Education, China (15YJC790074) and the PhD Start-up Fund of Natural Science Foundation of Guangdong Province, China (2014A030310305).

\section{REFERENCES}

[1] Black, F. \& Scholes, M. (1973),The Pricing of Options and Corporate Liabilities. Journal of political economy, 81, 637-654;

[2] Duffie, D. \& Singleton, K. J. (1999), Modeling Term Structures of Default Able Bonds. The review of financial studies, 12, 687-720;

[3] Erdelyi, A., Magnus, W., Oberhettinger, F. \&Tricomi, F. G. (1954), Tables of Integral Transforms: Vol.: 1-2. McGraw-Hill Book Company, Incorporated; [4] Gzyl, H., Milev, M. \&Tagliani, A. (2017), Discontinuous Payoff Option Pricing by Mellin Transform: A Probabilistic approach. Finance Research Letters, 20,281-288;

[5] Hull, J. \& White, A. (1995), The Impact of Default Risk on the Prices of Options and other Derivative Securities. Journal of Banking \& Finance, 19, 299-322;

[6] Jarrow, R. A., Lando, D. \& Turnbull, S. M. (1997), A Markov Model for The Term Structure of Credit Risk Spreads. The review of financial studies, 10, 481-523;

[7] Jarrow, R. A. \& Turnbull, S. M. (1995), Pricing Derivatives on Financial Securities Subject to Credit Risk. The journal of finance, 50, 53-85; [8]Jeon, J. \& Kim, G. (2019), Pricing of Vulnerable Options with Early Counter Party Credit Risk. The North American Journal of Economics and Finance, 47, 645-656; 
[9] Jeon, J., Yoon, J.-H. \& Kang, M. (2017),Pricing Vulnerable Path-dependent Options Using Integral Transforms. Journal of Computational and Applied Mathematics, 313, 259-272;

[10] Johnson, H. \& Stulz, R. (1987),The Pricing of Options with Default Risk. The Journal of Finance, 42, 267-280;

[11] Kim, G. \& Koo, E. (2016),Closed-form Pricing Formula for Exchange Option with Credit Risk. Chaos, Solitons \& Fractals, 91, 221-227;

[12] Lando, D. (1998), On Cox Processes and Credit Risky Securities. Review of Derivatives research, 2, 99-120;

[13] LI, T. R. \& Rodrigo, M. R. (2017), Alternative Results for Option Pricing and Implied Volatility in Jump-Diffusion Models Using Mellin Transforms.

European Journal of Applied Mathematics, 28, 789-826;

[14] Longstaff, F. \& Schwartz, E. (1995), Valuing Credit Derivatives. Journal of Fixed Income, 5, 6-12;

[15] Merton, R. C. (1974), On the Pricing of Corporate Debt: The Risk Structure of Interest Rates. The Journal of finance, 29, 449-470;

[16] Panini, R. \&Srivastav, R. P. (2004), Option Pricing with Mellin Transforms. Mathematical and Computer Modelling, 40, 43-56;

[17] Rizal, N. A.,Wiryono, S. K. \&Prasetyo, A. D. (2018),Optimal Assets Allocation for Risk Averse Investor under Market Risks and Credit Risk. International Journal of Business and Society, 19, 245-258;

[18] Sneddon, I. N. (1972), The Use of Integral Transforms. McGraw-Hill; [19] Lee, Min-Ku, Yang, Sung-Jin \& Kim,Jeong-Hoon, (2017),Pricing Vulnerable Options with Constant Elasticity of Variance versus Stochastic Elasticity of Variance. Economic Computation \& Economic Cybernetics Studies \& Research; ASE Publishing; 51(1), 233-247;

[20] Wang, X. (2018), Pricing Vulnerable European Options with Stochastic Correlation. Probability in the Engineering and Informational Sciences, 32, 67-95; [21] Yoon, J.-H. \& Kim, J.-H. (2015), The Pricing of Vulnerable Options with Double Mellin Transforms. Journal of Mathematical Analysis and Applications, $422,838-857$. 